\title{
Structural characterization of coatomer in its cytosolic state
}

\author{
Shengliu Wang ${ }^{1,4}$, Yujia Zhai ${ }^{1}$, Xiaoyun Pang ${ }^{1}$, Tongxin Niu ${ }^{1,4}$, Yue-He Ding ${ }^{2}$, Meng-Qiu Dong ${ }^{2}$, \\ Victor W. Hsu ${ }^{5}$, Zhe Sun ${ }^{1 凶}$, Fei Sun ${ }^{1,3,4 \bowtie}$ (]) \\ ${ }^{1}$ National Key Laboratory of Biomacromolecules, Institute of Biophysics, Chinese Academy of Sciences, Beijing 100101, \\ China \\ ${ }^{2}$ National Institute of Biological Sciences, Beijing, Beijing 102206, China \\ ${ }^{3}$ Center for Biological Imaging, Institute of Biophysics, Chinese Academy of Sciences, Beijing 100101, China \\ ${ }^{4}$ University of Chinese Academy of Sciences, Beijing 100049, China \\ ${ }^{5}$ Department of Medicine, Harvard Medical School, Brigham and Women's Hospital, Boston, MA 02115, USA \\ $\triangle$ Correspondence: zsun@moon.ibp.ac.cn (Z. Sun), feisun@ibp.ac.cn (F. Sun)
}

Received May 7, 2016 Accepted June 23, 2016

\begin{abstract}
Studies on coat protein I (COPI) have contributed to a basic understanding of how coat proteins generate vesicles to initiate intracellular transport. The core component of the COPI complex is coatomer, which is a multimeric complex that needs to be recruited from the cytosol to membrane in order to function in membrane bending and cargo sorting. Previous structural studies on the clathrin adaptors have found that membrane recruitment induces a large conformational change in promoting their role in cargo sorting. Here, pursuing negative-stain electron microscopy coupled with singleparticle analyses, and also performing CXMS (chemical cross-linking coupled with mass spectrometry) for validation, we have reconstructed the structure of coatomer in its soluble form. When compared to the previously elucidated structure of coatomer in its membrane-bound form we do not observe a large conformational change. Thus, the result uncovers a key difference between how COPI versus clathrin coats are regulated by membrane recruitment.
\end{abstract}

KEYWORDS coatomer, COPI, human, single-particle electron microscopy, membrane trafficking

Shengliu Wang and Yujia Zhai have made equal contributions to this work.

Electronic supplementary material The online version of this article (doi:10.1007/s13238-016-0296-z) contains supplementary material, which is available to authorized users.

\section{INTRODUCTION}

Coat proteins play a key role in intracellular transport by initiating the formation of transport vesicles. They achieve this role by coupling two major functions, bending membrane that generates transport carriers from organellar membrane, and binding to cargoes for the proper packaging of newly formed vesicles. The ADP-ribosylation factor (ARF) family of small GTPases has been shown to regulate these function of coat proteins by dictating their recruitment from the cytosol to membrane (Bonifacino and Glick, 2004).

Studies on coat protein I (COPI) have contributed to a basic understanding of how coat proteins achieve its roles in vesicle formation and cargo sorting (Faini et al., 2013; Hsu et al., 2009; Jackson, 2014; Pucadyil and Schmid, 2009). The core component of the COPI complex is coatomer, which exists as a multimeric complex containing seven subunits: $\alpha-, \beta-, \beta^{\prime}-, \gamma^{-}, \delta-, \varepsilon-$, and $\zeta$-COP (Hara-Kuge et al., 1994; Malhotra et al., 1989; Waters et al., 1991). Large efforts have been made to elucidate the structure of coatomer over the years, including several crystal structures of fragments of the seven subunits (Jackson et al., 2012; Lee and Goldberg, 2010; Ma and Goldberg, 2013; Suckling et al., 2015; Yu et al., 2012), and electron microscopy (EM)-based reconstruction of the entire structure of coatomer (Dodonova et al., 2015; Yip and Walz, 2011).

Coatomer can be further divided into two subcomplexes, with the B-subcomplex containing $\alpha, \beta^{\prime}$, and $\varepsilon$ subunits and the F-subcomplex containing $\beta, \delta, y$, and $\zeta$ subunits. The F-subcomplex shows significant structural similarity to the clathrin adaptors and interacts with two molecules of 
activated (GTP-bound) form of ARF1 (ADP-ribosylation factor 1) via the $N$-termini of $\beta$ - and $\gamma$-COP (Faini et al., 2013; Serafini et al., 1991b; Yu et al., 2012). In the B-subcomplex, $\alpha-$ and $\beta^{\prime}$-COP are considered structural homologs. Both adopt the domain structure of $\beta$-propeller- $\beta$-propeller- $\alpha$ solenoid, and they form a heterodimer via the $\alpha$-solenoid region (Lee and Goldberg, 2010).

In recent years, structural studies on the clathrin adaptors have shed important additional insights into how membrane recruitment regulates the function of coat proteins. Clathrin AP2 has been found to undergo a major conformational change upon its recruitment from the cytosol to membrane (Jackson et al., 2010). This change is instigated by AP2 binding to a key lipid, phosphatidylinositol $(4,5)$ bis-phosphate (PIP2), at the plasma membrane. The soluble form of AP2 exists in a "closed" conformation, which prevents cargo binding by AP2 (Collins et al., 2002). Binding to PIP2 on membrane induces an "open" conformation of AP2 to allow cargo binding (Jackson et al., 2010). Structural study on clathrin AP1 also suggests that it undergoes a major conformational change upon membrane recruitment (Ren et al., 2013). In the soluble state, AP1 adopts a similarly "closed" conformation to prevent cargo binding (Heldwein et al., 2004). Upon binding to ARF1 on membrane, AP1 shifts into an "open" conformation to allow cargo binding (Ren et al., 2013).

As basic mechanisms of intracellular transport are generally conserved (Bonifacino and Glick, 2004), coatomer is predicted to undergo a similar large conformational change during membrane recruitment. However, recent structural reconstructions of membrane-bound coatomer have begun to identify key differences between how COPI and the clathrin complexes are organized on membrane for vesicle formation (Dodonova et al., 2015; Faini et al., 2012). As such, whether the large conformational changes that have been observed for the membrane recruitment of clathrin adaptors would also apply to the COPI complex becomes an open question. In this study, we address this key mechanistic issue by elucidating the structure of coatomer in its soluble form. By comparing this structure with the previously solved structure of coatomer in its membrane-bound form (Dodonova et al., 2015), we have uncovered yet another key difference between coatomer and the clathrin adaptors.

\section{RESULTS}

\section{Expression and purification of recombinant human coatomer}

Coatomer exists as a single complex of approximately $560 \mathrm{kDa}$ (Waters et al., 1991). The subunits of coatomer and their major domains are shown in Fig. 1A. To facilitate the isolation of highly purified coatomer for structural analysis, recent studies have generated recombinant mouse coatomer using a MultiBac expression system (Dodonova et al., 2015; Faini et al., 2012; Sahlmuller et al., 2011). In the present study, we developed a novel protein complex expression system, ViperTEC (to be published elsewhere), to express recombinant human coatomer in insect cells. Specifically, cDNAs of $\alpha-, \beta^{\prime}-$, and $\varepsilon$-COP were inserted into one expression cassette containing a p6.9 promoter and a SV40 terminator, while cDNAs for the other four subunits ( $\beta$-, $\mathrm{Y}-, \delta$-, and $\zeta$-COP) were inserted into another expression cassette containing a p10 promoter and a SV40 terminator. Based on previous structural studies (Jackson et al., 2012; Lee and Goldberg, 2010; Ma and Goldberg, 2013; Suckling et al., 2015; Yu et al., 2012), tags were introduced at the 5' end of each subunit to minimize potential effects on the subsequent structure and function of coatomer. As will be seen below, this has facilitated our ability to track the expression of individual subunits and their assembly state during purification. Moreover, we did not detect significant effects on coatomer function.

To purify the recombinant human coatomer expressed in insect cells, we performed Strep tag II affinity chromatography followed by Mono $Q$ anion exchange chromatography. Multiple observations suggested that this approach resulted in intact coatomer being purified. First, Coomassie-stained SDS-PAGE gel showed the presence of all seven subunits of coatomer (Fig. 1B), which was further verified by Western blotting (Fig. S1). Second, gel filtration showed a single peak during elution (Fig. 1C). Third, native PAGE reveals a monodisperse band, indicating that the recombinant coatomer was isolated with high purity and homogeneity (Fig. 1D). Fourth, analysis using liquid chromatography mass spectrometry (LC-MS) of the single band observed in native page resulted in all seven subunits of coatomer being identified (Table 1). Our yield of purified coatomer was approximately $1.5 \mathrm{mg}$ per liter of cell culture.

Our previous experiences in purifying native coatomer from tissue suggested that the linkage between the $\mu$-homology domain and the $\mathrm{N}$-terminal domain (NTD) of $\delta$-COP is flexible, as the $\mu$-homology domain was frequently degraded (unpublished data). In the purification of recombinant coatomer, we also observed the degradation of $\delta$-COP, which appeared as an additional band in Coomassie-stained gel (Fig. 1B). Western blotting (Fig. S1) and mass spectrometry analysis (Table S1) identified this additional band as the N-terminal portion of $\delta$-COP. Collectively, these observations suggested that a portion of $\delta$-COP is likely to be highly exposed on the surface of coatomer, as this portion shows consistent partial degradation during the purification of coatomer.

\section{In vitro functional assays of the recombinant human coatomer}

We next assessed whether the purified recombinant human coatomer is functional. For this goal, we generated recombinant human N-myristoylated ARF1 (Fig. S2A). Moreover, we used liposomal membrane rather than Golgi membrane, 
A
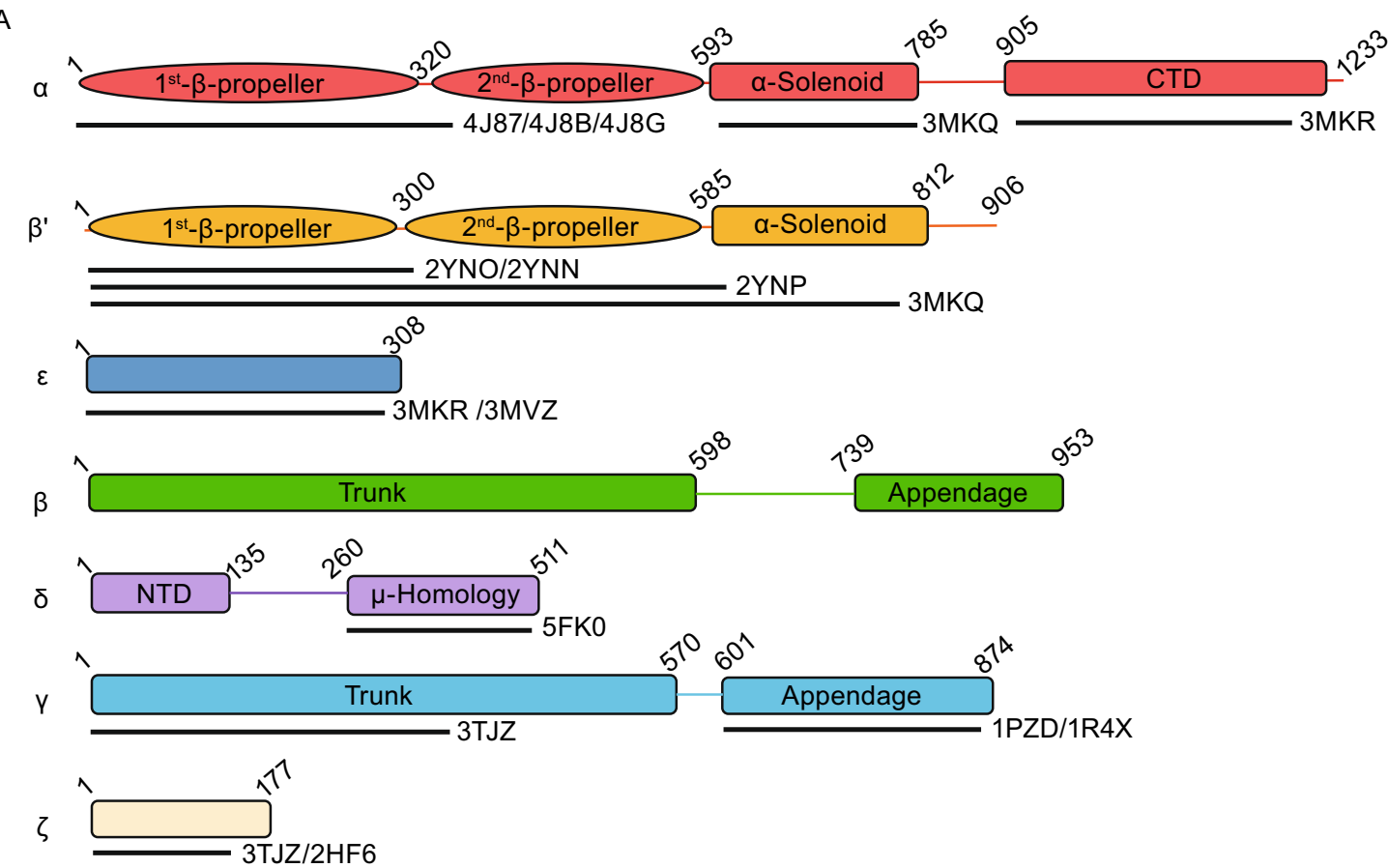

B

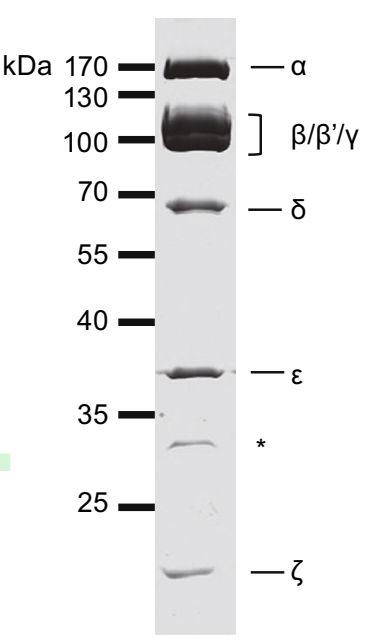

C

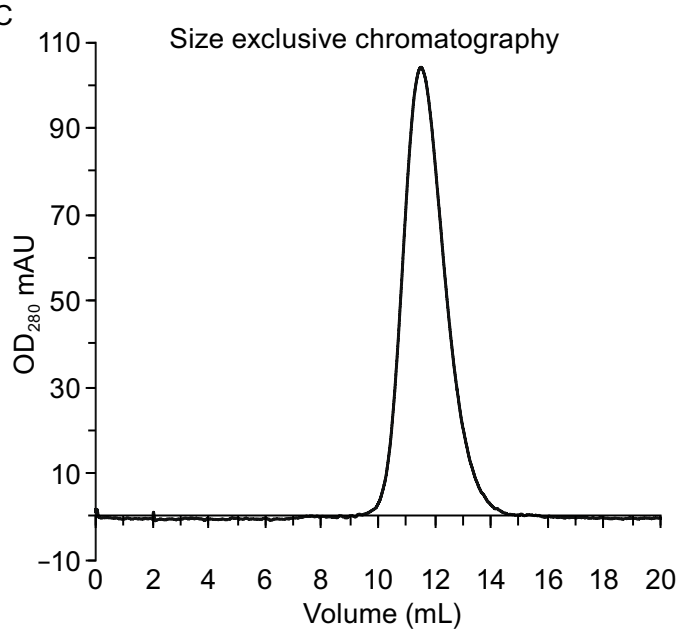

D

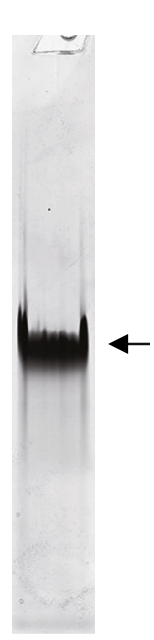

Figure 1. Preparation of purified recombinant human coatomer. (A) Schematic diagram showing the domain organization of each subunits of human coatomer. The available atomic structures with their PDB IDs and sequence coverage are also labeled. (B) Coomassie-blue stained SDS-PAGE of recombinant coatomer with all the subunits labeled. Asterisk indicates the N-terminal fragment of $\delta$-COP, which is confirmed by mass spectrometry and Western blot. (C) Gel filtration of purified coatomer. The retention volume of the single peak on the column of Superose 6 (GE healthcare) is $11.4 \mathrm{~mL}$. (D) Native PAGE of the purified coatomer. Arrow indicates the intact coatomer.

as native membrane contains a myriad of other proteins that can confound the ability to assess directly the roles of ARF1 and coatomer in COPI vesicle formation. When recombinant forms of ARF1 and coatomer were incubated with liposomes that contain a Golgi-like composition of pure lipids, we confirmed that ARF1 enhanced the recruitment of coatomer onto membrane (Fig. 2A). We also confirmed that the GTP-bound form of ARF1 was more efficient in binding to liposomes and in recruiting coatomer to this membrane (Fig. 2A). Thus, the recombinant forms of ARF1 and coatomer that we had generated are functional with respect to membrane recruitment.

We next considered that the GTPase-activating protein (GAP) responsible for catalyzing the deactivation of ARF1 
Table 1. Mass spectrometry analysis of purified coatomer extracted from the band of native PAGE

\begin{tabular}{llcl}
\hline Subunit & Molecular mass $(\mathrm{kDa})$ & Unique peptide $(\mathrm{n})$ & Coverage $(\%)$ \\
\hline$\alpha-$ COP & 139 & 108 & 77.5 \\
$\beta-$ COP & 107 & 84 & 68.5 \\
$\beta^{\prime}-$ COP & 102 & 64 & 74.2 \\
Y-COP & 98 & 69 & 74.6 \\
$\delta$-COP & 57 & 33 & 54.2 \\
$\varepsilon-$ COP & 34 & 22 & 83.4 \\
$\zeta-$ COP & 20 & 6 & 37.9 \\
\hline
\end{tabular}

during COPI vesicle formation is ARFGAP1 (Cukierman et al., 1995; Yang et al., 2002). Thus, we also purified the recombinant form of ARFGAP1 (Fig. S2B). Consistent with previous studies that have found ARFGAP1 to induce the release ARF1 from Golgi membrane (Cukierman et al., 1995; Yang et al., 2002), we found that our recombinant ARFGAP1 also reduced the level of ARF1 on liposomal membrane (Fig. 2B). This effect of ARFGAP1 could be attributed to its catalytic activity, as the use of GTPYS (a non-hydrolyzable GTP analogue) prevented the ability of ARFGAP1 to reduce the level of ARF1 on liposomal membrane (Fig. 2B).

We next examined the effect of recruiting different combinations of coatomer, ARF1, and ARFGAP1 on membrane morphogenesis. ARF1 has been found previously to induce membrane curvature by inserting its $\mathrm{N}$-terminal amphipathic helix into the membrane, which is reflected by the generation of tubules that sometimes also contain constrictions, resulting in a "beads-on-string" morphology (Beck et al., 2008; Krauss et al., 2008; Lundmark et al., 2008). We observed similar effects when recombinant ARF1 was incubated with Golgi-like liposomes (Fig. 2C). As the co-incubation of activated ARF1 and coatomer has been found previously to be sufficient to produce COPI vesicles from liposomal membrane (Bremser et al., 1999; Spang et al., 1998), we also confirmed this finding using the recombinant forms of ARF1 and coatomer that we had generated (Fig. 2C). Further scrutiny of these vesicles revealed that they are coated (Fig. S3A) and have diameter in the range of $50-80 \mathrm{~nm}$ (Fig. 3). Western blotting confirmed the presence of ARF1 and $\beta$-COP on these vesicles (Fig. S3B).

We also noted that COPI vesicles reconstituted from Golgi membrane have been found to be relatively depleted of cholesterol (Brugger et al., 2000). Consistent with this previous finding, we found that liposomal membrane was more readily vesiculated with lower concentrations of cholesterol (Fig. S4A, and also Supplemental text). The diameters of vesicles were also affected by the level of cholesterol (Fig. S4B, and also Supplemental text).

Besides ARF1 and coatomer, ARFGAP1 has been found previously to promote COPI vesicle formation (Yang et al., 2002). Consistent with this finding, we observed vesiculation when ARFGAP1 was added in conjunction with ARF1 and coatomer to liposomes (Fig. 2C). In this case, vesicles of smaller diameter (in the range of $30 \mathrm{~nm}$ ) were observed (Fig. 2C). Similar to ARF1, ARFGAP1 is also known to possess a domain that can insert into the membrane (Bigay et al., 2003). Thus, a likely explanation is that the combination of ARF1, coatomer, and ARFGAP1 results in greater membrane curvature being generated, which is manifested by the formation of smaller vesicles.

We also found that coatomer alone could be recruited onto liposomal membrane (Fig. 2B), with EM examination revealing that this condition resulted in liposome tubulation (Fig. 2C). To our knowledge, this finding revealed a property of coatomer that had not been widely appreciated. Future studies will be needed to elucidate more precisely how coatomer exerts this effect. In any case, as the cumulative results above represent substantial support that the recombinant form of human coatomer that we have generated is functional, we next pursued structural analysis of this coatomer in its soluble form.

\section{Electron microscopy of coatomer in its soluble form}

We initially pursued cryo-electron microscopy but failed to obtain high-quality micrographs, which could be attributed to coatomer being prone to collapse after cryo-vitrification, as the air-water interface that occurs in this procedure could produce damaging effects. Thus, we next pursued negativestain electron microscopy coupled with computational image-processing methods.

We detected soluble coatomer as mono-dispersed particles with diameter in the range of 13-20 nm (Fig. S5A). These were selected for further image processing. Using reference-free 2D classification, we found that class averaging of most particles revealed a central density that is relatively invariant (Fig. S5B), while more peripheral densities appear variable in both location and shape (Fig. S5B). These findings were consistent with previous observations on the structure of yeast coatomer (Yip and Walz, 2011). Further scrutiny of the central density revealed that it exists as two parts (Fig. S5B and S5C). We also deduced that the variable extra densities could be attributed to flexible domains known to exist in coatomer. Besides $\delta$-COP, these would include portions of the $\alpha^{\mathrm{CTD}} / \varepsilon$-COP heterodimer and the appendage domains of $\beta / \gamma-C O P$. 
A

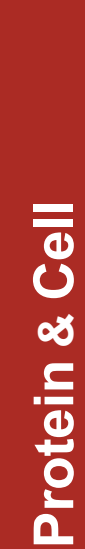

C

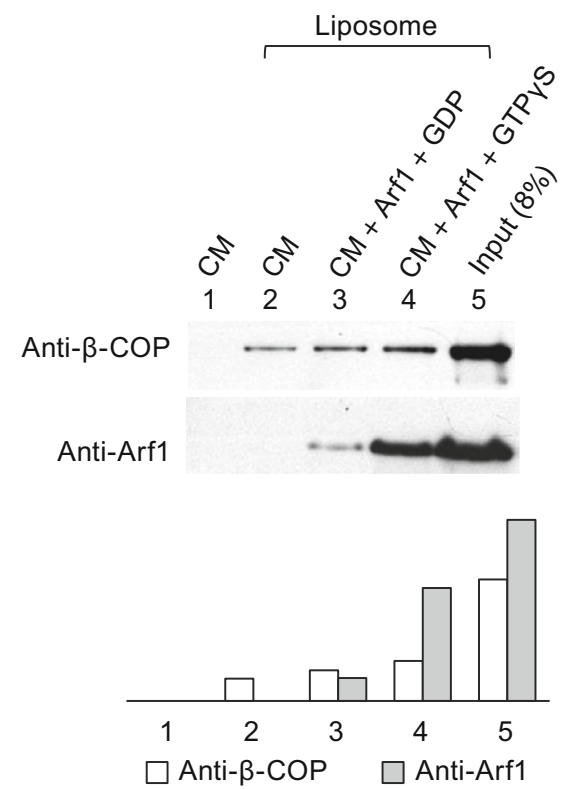

B
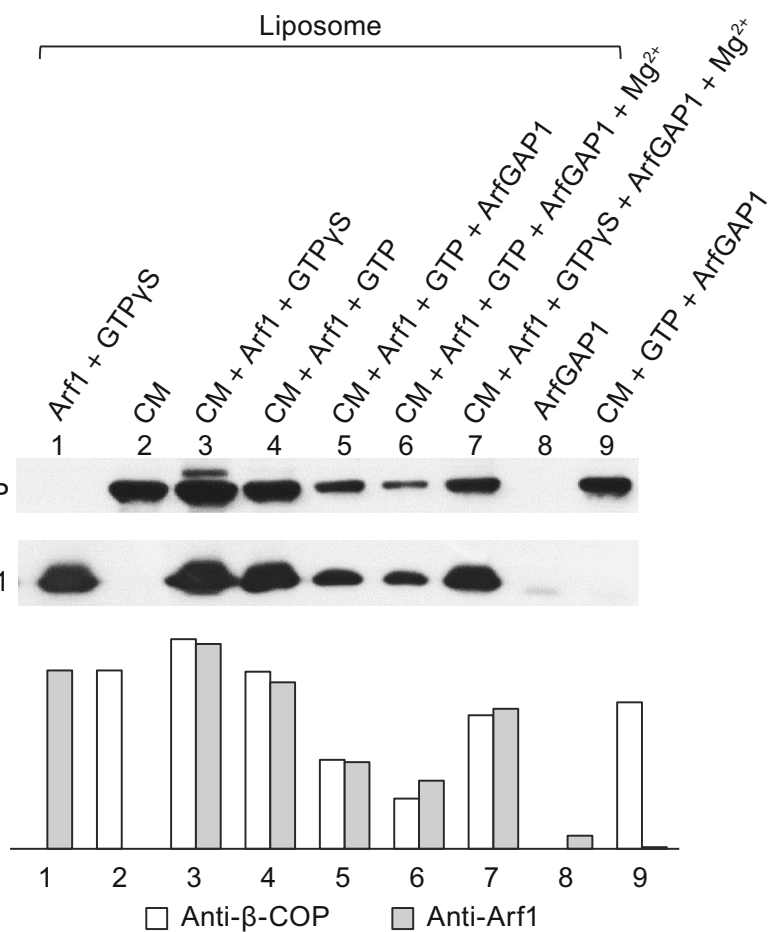
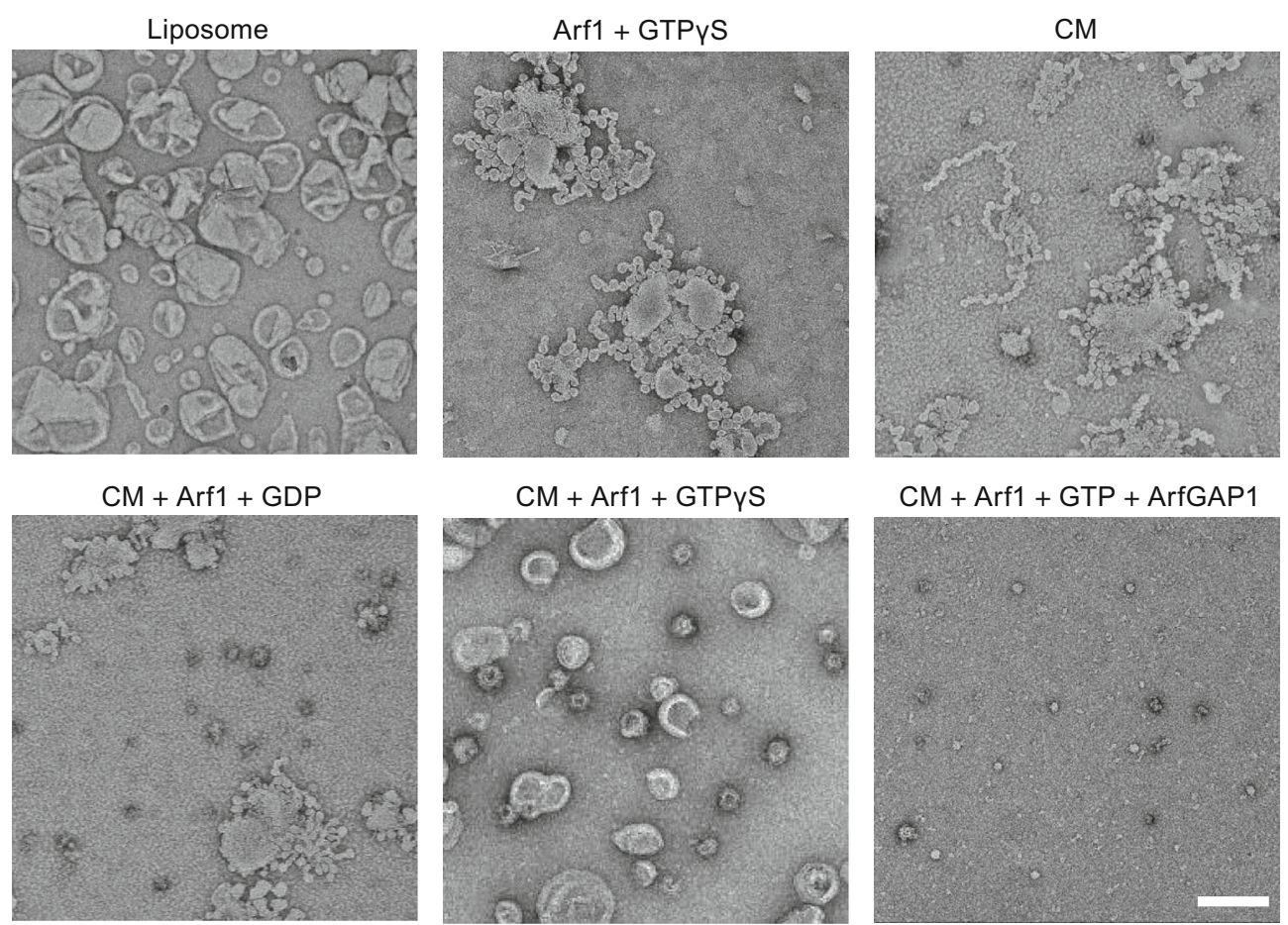

Figure 2. Membrane binding and vesiculation assays. ( $A$ and $B$ ) Membrane binding co-sedimentation assays (See MATERIALS AND METHODS). The amount of co-sedimented coatomer ( $\beta$-COP) and ARF1 is also quantified in lower panels. (C) Negative-stain electron microscopy of liposomes incubated with coatomer and other factors. Scale bar, $200 \mathrm{~nm}$. CM, human coatomer; ARF1, $\mathrm{N}$-myristoylated human ARF1; ARFGAP1, rat ARFGAP1. 
We then pursued single-particle analysis. To avoid potential modeling bias, we collected the tilt series of negatively stained coatomer and performed electron tomographic reconstruction followed by sub-tomogram averaging to generate a reliable model. We performed intensive iterative 2D and 3D classifications to sort the particle sets. We filtered out overlapping, imperfectly stained, or disrupted particles. The final reconstruction based on the filtered dataset yielded a 3D structure of coatomer with a resolution of $19 \AA$, as judged by Fourier shell correlation (FSC) criterion of 0.143 (Rosenthal and Henderson, 2003) (Figs. 4A and S5). Consistent with the results from the $2 \mathrm{D}$ class averages, the overall reconstructed EM map revealed soluble coatomer to possess two relatively independent parts. One part (part 1) adopts an arch shape (Fig. 4A, pink), while another part (part 2) sits adjacent to the arch-shaped structure and appears more globular (Fig. 4A, yellow). The arch-shaped density (part 1) was invariant during iterative $3 D$ classifications, while the globular density (part 2) varied in different cycles of 3D classifications.

As the two parts of coatomer appeared to behave differently and independently, we next pursued local optimization (Shan et al., 2016) and performed "focus" refinement (Bai et al., 2015) to reconstruct the two parts separately (Fig. S6A; see also "MATERIALS AND METHODS"). The "focus" refinement resulted in a similar density map for the arch-shaped portion (part 1), and a different density map for the globular portion (part 2) (Fig. S6A). The final resolution was $18 \AA$ and $20 \AA$, respectively, with FSC of 0.143 (Rosenthal and Henderson, 2003) (Fig. S6B). There was a flexible oscillation between the two parts, and the alignment procedure preferred the more stable part1 during the conventional refinement (Figs. $4 \mathrm{C}$ and S5A).

The recently solved structure of membrane-bound coatomer (Dodonova et al., 2015) provided an opportunity to analyze and interpret the EM maps of the soluble coatomer that we have generated. In order to detect potential structural difference between the two states, we initially fitted the complete structural model of the membrane-bound coatomer (PDB ID: 5A1U) as a rigid body onto the integrated density map before the "focus" refinement using UCSF Chimera (Pettersen et al., 2004). A good match was observed between the $\alpha / \beta^{\prime}-C O P$ heterodimer and the arch-shaped density. Thus, we assigned the arch-shaped density to correspond to the B-subcomplex of coatomer (Fig. 4B). In further support of this assignment, we noted that the $\alpha / \beta^{\prime}$-COP heterodimer has been suggested to be rigid, showing little difference when comparing the structure in solution (Lee and Goldberg, 2010) versus that on membrane (Dodonova et al., 2015). Thus, when taken collectively, we also assigned the other part of coatomer that appears as a globular density to correspond to the F-subcomplex. However, the fit in this case was relatively weak (Fig. 4B). This could be attributed to conformational changes of F-subcomplex between two states, or the potential flexible oscillation between the two subcomplexes of coatomer.

With the improved density map after "focus" classification and refinement, we also compared our reconstructed F-subcomplex with the three crystal structures of clathrin AP1 (the F-subcomplex homologue) in different conformations as well as the structure of membrane-bound F-subcomplex in the "hyper-open" state (Dodonova et al., 2015) to fit into the focus-refined density map. Structural models of AP1 included its "hyper-unlocked" state (PDB ID: 4P6Z) (Jia et al., 2014), "unlocked" state (PDB ID: 4HMY) (Ren et al., 2013) and "locked" state (PDB ID: 1W63) (Heldwein et al., 2004). As the C-terminal $\mu$-homology domain of $\delta$-COP is prone to degradation, and also considering that this domain was omitted in the previous reconstruction of membranebound coatomer, we omitted this domain during the fitting. The appendage domains of $\beta$ - and $\mathrm{\gamma}$-COP were also omitted, as they are known to be linked flexibly to the trunk domains. After incorporating all these considerations, we found that the "hyper-open" conformation of F-subcomplex gave the best fit (Fig. 4D). Besides the densities occupied by the F-subcomplex, the unassigned density located underneath
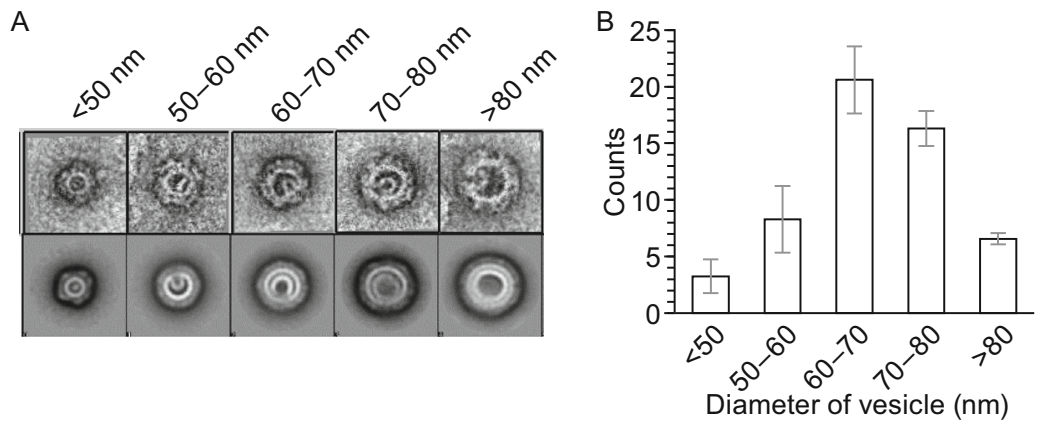

Figure 3. Diameter distribution of reconstituted COPI-coated vesicles. (A) The raw images of vesicles (first row) and the corresponding $2 \mathrm{D}$ class averages of vesicles with the same diameter (second row). The diameters are indicated on the top. (B) $A$ statistical histogram of the vesicle number vs. different diameters. All error bars represent standard deviation (s.d.) from three independent vesicle reconstitution experiments. For each experiment, the number $(\mathrm{N})$ of micrographs is 90 . 
A
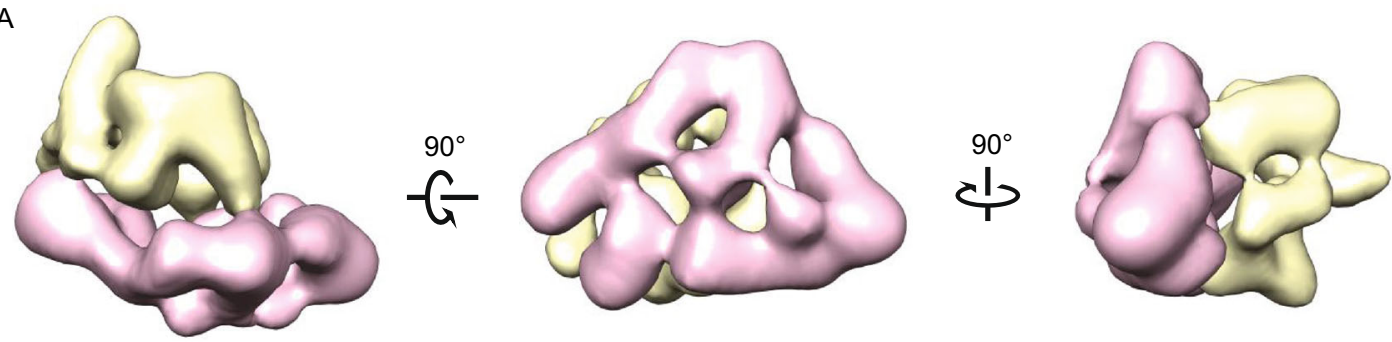

B
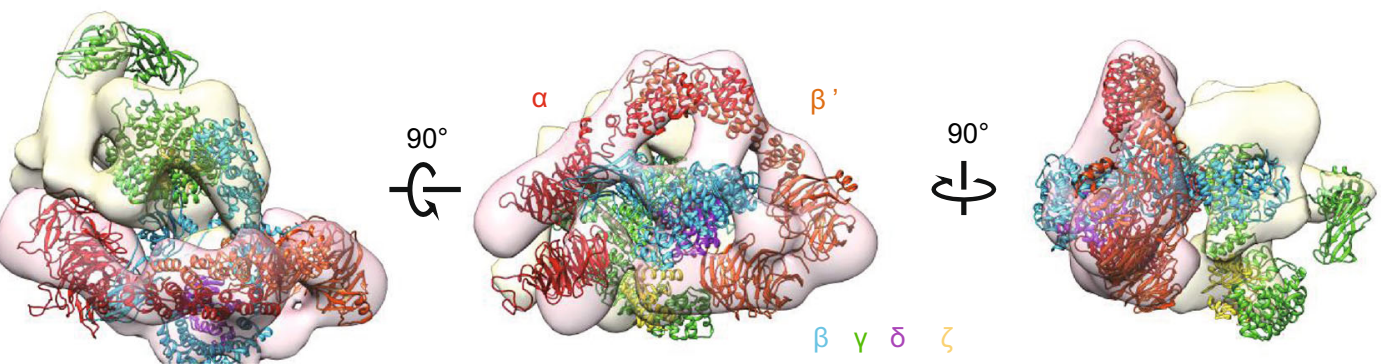

C $\alpha / \beta$ '-COP "arch"
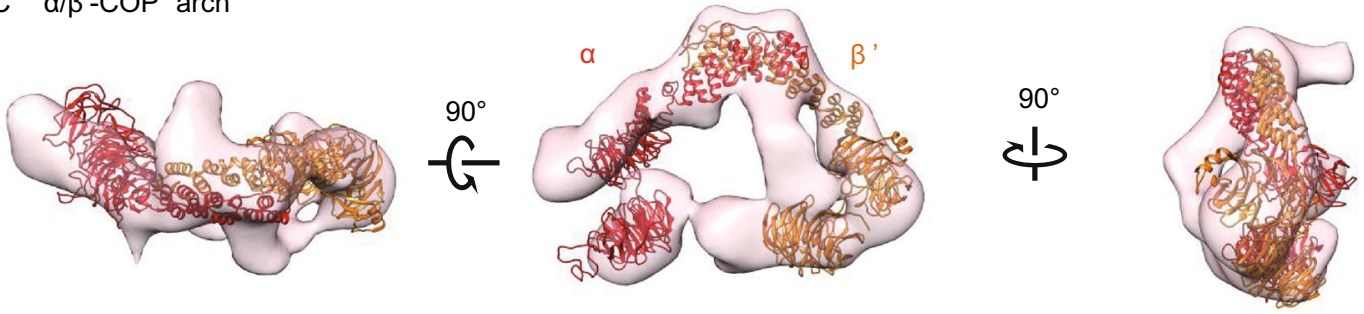

D $\beta \delta / y \zeta-C O P$ "Hyper-open"
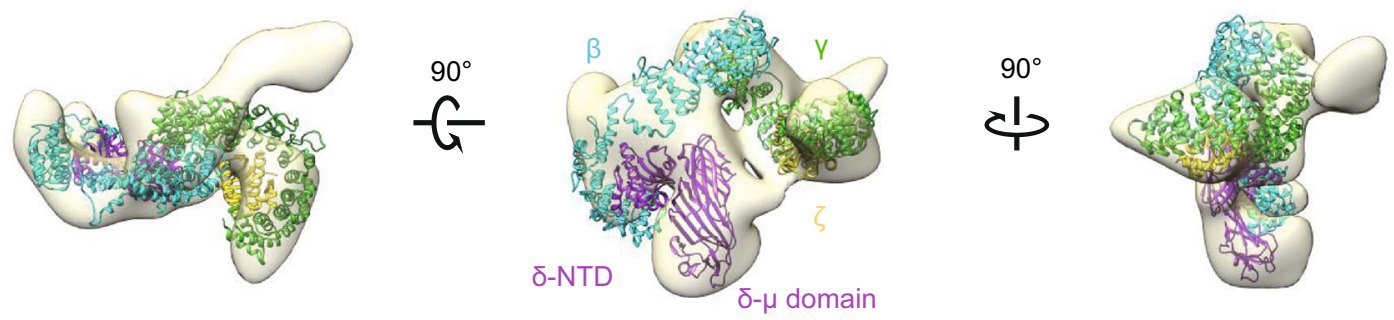

E Clathrin AP-1"Hyper-unlocked"
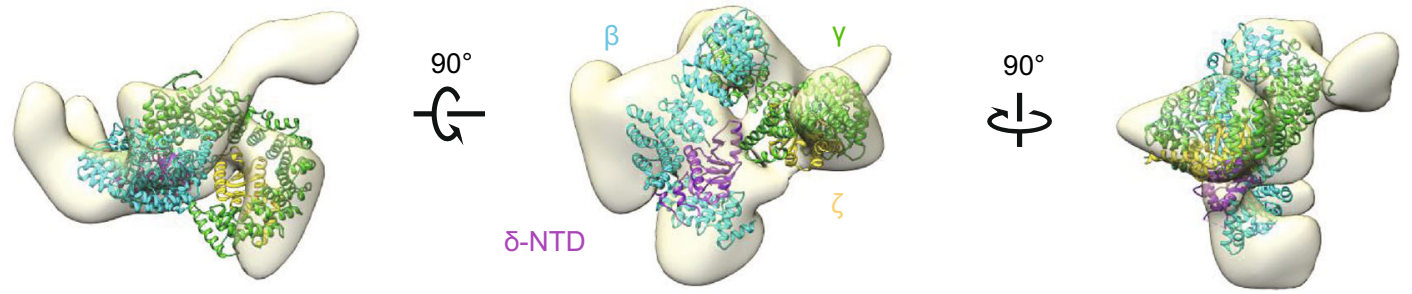

Figure 4. 3D reconstruction of the recombinant human coatomer in its soluble form. (A) Three views of surface-rendered density map of coatomer obtained by single-particle reconstruction. The map is segmented using UCSF Chimera, grouped, and colored with pink for the arch-shape portion and yellow for the more globular portion. (B) Fitting the membrane-bound model of coatomer (PDB ID: 5A1U) into the EM map. (C) Fitting the B-subcomplex $\alpha \beta^{\prime}$-COP heterodimer into the "focus" refined arch-shape density map. (D) Fitting the F-subcomplex $\beta \delta / y \zeta-C O P$ into the "focus" refined globular density map. (E) Fitting the homologue model of F-subcomplex in "hyper-unlocked" form into the "focus" refined globular density map. $\alpha-, \beta$ '-COP are colored in red and orange. $\beta$-, $\delta-, \gamma^{-}$, and $\zeta-C O P$ and their homologues are colored in cyan, purple, green, and yellow respectively. 
the "arch" on the $\beta / Y$-COP side could be assigned to the $C$-terminal $\mu$-homology domain of $\delta$-COP with good fit (Fig. 4D). As for the three structural models of AP1, only the "hyper-unlocked" conformation was found to give a reasonable fit (Fig. 4E). Furthermore, to test the possibility that the F-subcomplex can exist in multiple conformations, we performed 3D classification. However, after several cycles of classifications, we still could not detect any other conformation (Fig. S7).

Thus, the collective findings led us to conclude that the F-subcomplex exhibits an open conformation when coatomer is in its soluble form. Moreover, this conformation is similar to that seen previously for the membrane-bound form of coatomer (Dodonova et al., 2015). We further considered that the negative-stain EM approach provides a low-resolution structure of soluble coatomer. Thus, when also considering that flexible oscillation exists between the F-subcomplex and B-subcomplex, we did not attempt to join the two parts of structural reconstruction into one complete model of coatomer.

\section{CXMS analysis of coatomer in its cytosolic state}

We next sought to further validate the structural reconstruction of coatomer we had generated above by taking another independent approach. Chemical cross-linking of proteins coupled with mass spectrometry (CXMS) has been developed in recent years as a way of validating structural models (Leitner et al., 2014; Yang et al., 2012). We found that there were 82 pairs of DSS or $\mathrm{BS}^{3}$ cross-linked lysine residues that could be detected with high confidence in the soluble form of coatomer, of which 50 were within the same domain and the remaining 32 occurred as cross-links between different domains. Among these inter-domain cross-links, 14 occurred within the subcomplexes of coatomer, while 18 occurred as links between the two subcomplexes (Table S2).

We next mapped all the CXMS pairs into the model of the previously elucidated membrane-bound coatomer and measured the $\mathrm{C} \alpha-\mathrm{C} \alpha$ distances between the cross-linked residues (Table S2). The generally accepted $\mathrm{C} \alpha-\mathrm{C} \alpha$ distance between two cross-linked lysine residues, when using DSS or $\mathrm{BS}^{3}$ as a cross-linker, is below $\sim 35 \AA$ (Politis et al., 2014). Since the model of the membrane-bound coatomer was generated from a $13-\AA$ cryo-EM map (Dodonova et al., 2015), we relaxed the distance restriction of $\mathrm{Ca}-\mathrm{Ca}$ to $45 \AA$. This resulted in all the intra-domain cross-links having $\mathrm{Ca}$ $\mathrm{C} \alpha$ distances below $30 \AA$ except for one pair that was below $35 \AA$ (Table S2 and Fig. 5A). These findings suggested that the conformation of individual domains changes very little from the soluble to the membrane-bound form of coatomer.

As for the inter-domain cross-links, we found that 11 had distances greater than the cut-off value of $45 \AA$. Ten of these links were located between $\alpha^{\mathrm{NTD}}-\mathrm{COP}$ and the $\alpha^{\mathrm{CTD}} / \varepsilon-\mathrm{COP}$ heterodimer (Table S2 and Fig. 5A, orange). Furthermore, the cross-link distances between $\alpha^{\mathrm{CTD}}$-COP and $\varepsilon$-COP were below the cut-off value (Table S2). Thus, these results suggested that the heterodimer of $\alpha^{\mathrm{CTD}} / \varepsilon$-COP is structurally stable but changes its location between the soluble and membrane-bound forms of coatomer. In the soluble form, the

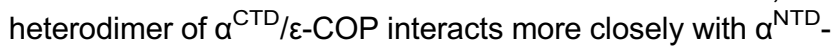
COP. The 11th cross-link that was observed to have greater distance than the cut off value resides between the appendage domain of $\beta$-COP and the $\mathrm{N}$-terminal domain of $\delta$-COP (Table S2 and Fig. 5A). These two domains were far from each other in the membrane-bound form of coatomer, but were sufficiently close in the soluble form of coatomer to become cross-linked, thus suggesting a conformational change. Since the appendage domain of $\beta$-COP interacts with the $\mathrm{N}$-terminal $\beta$-propeller domains of $\alpha$-COP and contributes to the interaction between the two subcomplexes of coatomer in the membrane-bound state, we speculated that the conformational changes predicted by the crosslinking approach corresponds to the flexible oscillation between two subcomplexes of coatomer in its soluble form. Aside from the cross-links described above, all other inter-domain links were below the cut-off value of $45 \AA$. Thus, the CXMS approach also suggested that coatomer does not undergo a large conformational change when comparing its soluble versus membrane-bound form.

We also sought to validate our fitting results above on the F-subcomplex of coatomer by examining all 16 pairs of cross-linked residues within the main structure of the F-subcomplex. To do this, we removed from consideration the known flexible regions within this subcomplex, which are the appendage domains of $\beta / \mathrm{Y}-\mathrm{COP}$ and the $\mu$-homology domain of $\delta$-COP. The three structural models of AP1 in different conformations were used as reference, as well as the membrane-bound form of coatomer. We found that all the $\mathrm{C} \alpha-\mathrm{C} \alpha$ distances of the cross-links were below $35 \AA$ for the "hyper-open" coatomer F-subcomplex and below $40 \AA$ for the "hyper-unlocked" clathrin AP-1 (Fig. 5B and Table S3). However for the "unlocked" and the "locked" clathrin AP-1 models, there were some cross-links with larger distance than the cut-off value of $45 \AA$ (Fig. 5B and Table S3). Thus, these results suggested that the F-subcomplex exhibits a relatively open conformation in the soluble form of coatomer. Notably, this conclusion is also consistent with the result above derived from the rigid-body fitting of the negative-stain EM map (Fig. 4D and 4E).

\section{DISCUSSION}

Using negative-stain EM and single-particle analysis, we have reconstructed the 3D structure of recombinant human coatomer in its soluble form (Fig. 4). This structure has been further validated by an independent approach using CXMS analysis. We then compared our reconstructed structure of soluble coatomer with a previously reconstructed structure of membrane-bound coatomer to reach a key conclusion, which is that soluble coatomer adopts largely a similar overall conformation as the membrane-bound form. 

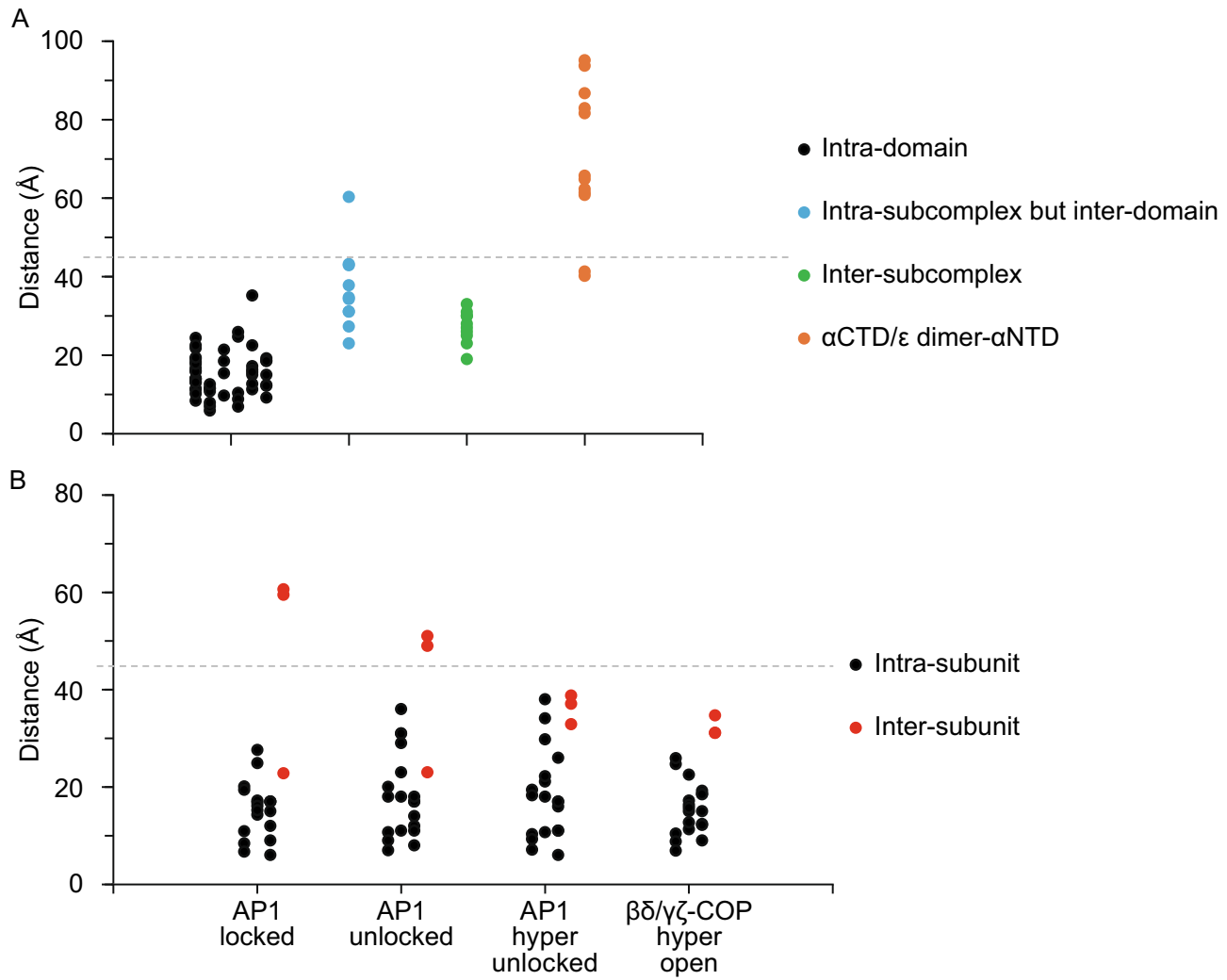

Figure 5. Mapping CXMS cross-linked residue pairs onto structural models. (A) The distances between Ca-Ca atoms of crosslinked residues pairs were measured based on the membrane-bound coatomer model (PDB ID: 5A1U), classified and plotted. (B) The distances between $\mathrm{Ca}-\mathrm{C} \alpha$ atoms of cross-linked residues pairs within the adaptor F-subcomplex were measured based on four models as indicated, classified into intra-subunit (black) and inter-subunit (red) ones and plotted. The cut-off distance of $45 \AA$ is represented by the dashed line.

Coatomer is comprised of two subcomplexes. We find that the B-subcomplex forms an arch-like structure, which does not vary significantly when comparing the soluble versus the membrane-bound form of coatomer (Fig. 4C). The F-subcomplex also appears largely similar when comparing the two states of coatomer. Moreover, when compared with the previously elucidated structures of clathrin AP1, the F-subcomplex adopts a conformation that is similar to the open conformation of AP1. Thus, in contrast to the case of clathrin adaptors, for which membrane recruitment induces a large conformational change from "closed" to "open" conformation, coatomer remains in the "open" conformation regardless of whether it resides in the cytosol or on membrane (Fig. 4D).

We further note that flexibility in coatomer has been noted previously (Langer et al., 2008; Reinhard et al., 1999; Yip and Walz, 2011). Our current results reveal that flexibility can come from the two subcomplexes of coatomer having mobile interactions. We also acknowledge that flexibility can come domains within individual subunits of coatomer. These would include $\delta$-COP, as well as portions of the $\alpha^{\mathrm{CTD} / \varepsilon-C O P}$ heterodimer and the appendage domain of $\beta / \mathrm{Y}-\mathrm{COP}$. However, due to the limited resolution of the negative-stain EM approach, a more detailed assessment of these flexibilities will require future studies that can achieve a higher-resolution structure of coatomer.

In any case, our current results suggest a speculative model for how coatomer is recruited onto membrane to participate in COPI vesicle formation (Fig. 6). Considering the flexible oscillation between the two subcomplexes of the coatomer, we propose that the interactions between membrane-anchored ARF1 and the F-subcomplex, together with the interaction between the scaffolding B-subcomplex and membrane, help to immobilize the interaction between the two subcomplexes of coatomer. This then triggers a conformational transition into the "active" form of coatomer on membrane. The subsequent self-assembly of coatomer, together with regions of ARF1 and coatomer interacting with the underlying membrane, induces curvature on this membrane, which eventually results in vesicle formation.

A new insight on coatomer has also come from our functional studies. Although early studies on COPI vesicle 


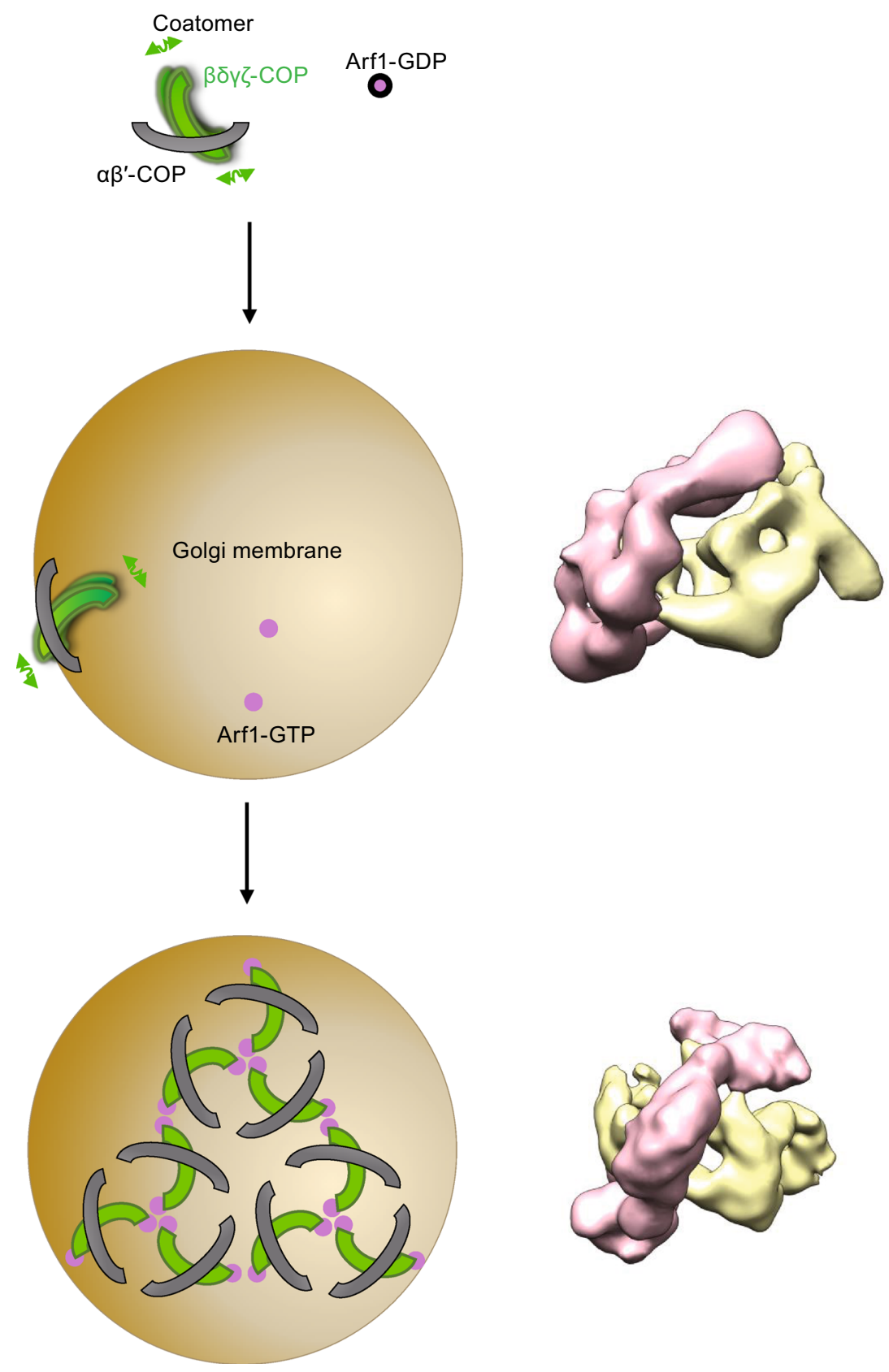

Figure 6. A working model for the membrane recruitment of coatomers in promoting COPI vesicle formation. The two subcomplexes (B-subcomplex in gray and F-subcomplex in green) of coatomer can oscillate in its cytosolic state. In the absence of ARF1-GTP (magenta), coatomer binds to membrane (brown) via the B-subcomplex, remodeling the membrane into tubules or "beads-on-string" morphology, for which the precise mechanism remains to be elucidated. Oscillation of the membrane-bound coatomer prevents its ability to drive vesicle formation. In the presence of membrane-anchored ARF1-GTP, the direct interaction between F-subcomplex and ARF1-GTP reduces the oscillation between the two subcomplexes, thereby driving the membrane-bound coatomer to a more stabilized (less oscillation) form. This stabilized coatomer is then able to assemble into oligomers, which together with ARF1, drives COPI vesicle formation.

formation have used Golgi membrane, the use of simpler liposomal membranes in recent years has provided a more direct way of assessing how key factors, such as coatomer and ARF1, act to achieve vesicle formation (Bremser et al.,
1999; Spang et al., 1998). In this regard, whereas ARF1 had been known to recruit coatomer from the cytosol to Golgi membrane in regulating the function of coatomer (Donaldson et al., 1992; Serafini et al., 1991a), the use of the simpler 
liposomal membrane has uncovered that ARF1 also possess a direct ability to induce membrane curvature (Beck et al., 2008; Krauss et al., 2008; Lundmark et al., 2008). In the current study, we have used of the simpler liposomal membrane to uncover that coatomer also possesses a direct ability to induce membrane curvature. To our knowledge, this behavior of coatomer has not been noted previously.

Studies on different coat complexes have revealed that single factors typically convert liposomes into tubules or "beads-on-string" morphology (Beck et al., 2008; Farsad et al., 2001; Lee et al., 2005a; Peter et al., 2004), while multiple factors are needed to convert liposomes into vesicles (Bremser et al., 1999; Matsuoka et al., 1998; Spang et al., 1998; Takei et al., 1998). We have observed similar effects, as the incubation of Golgi-like liposomes with either coatomer or ARF1 induces "beads-on-string" morphology, while their incubation together induces a more complete fission of liposomal membrane to generate COPI vesicles. These observations can be explained by the fission process requiring considerable force/strain being imposed on membrane. Thus, multiple factors acting in concert provide a way to generate such formidable force/strain.

At the mechanistic level, how protein factors induce membrane curvature is explained currently by two general mechanisms: i) introducing a wedge defect into one leaflet of the membrane bilayer that results in strain to promote curvature or ii) exerting a scaffolding mechanism that involves a curved protein structure binding to the underlying membrane through electrostatic interactions (McMahon and Gallop, 2005). The first mechanism explains how the ARF-like small GTPases induce membrane curvature, as they possess an $\mathrm{N}$-terminal amphipathic helix that can insert into one leaflet of the membrane bilayer to induce membrane curvature (Beck et al., 2008; Bielli et al., 2005; Hariri et al., 2014; Lee et al., 2005a). How coatomer induces membrane curvature is less clear. Based on the recent structural reconstruction of coatomer on membrane, four sites in coatomer are predicted to contact the underlying membrane (Dodonova et al., 2015). Two of them are located in F-subcomplex and interact with the underlying membrane indirectly via ARF1. The other two are located in the B-subcomplex, and can potentially interact directly with membrane. However, because a high-resolution structure for these potential direct binding sites is currently unavailable, the precise mechanism by which coatomer induces membrane curvature remains to be determined in the future.

It is also notable that, besides ARF1, coatomer has been found to interact with multiple other proteins upon recruitment to membrane. These include cargo proteins (Cosson and Letourneur, 1994; Fiedler et al., 1996) and the catalytic regulators of ARF1, its GEF (Deng et al., 2009), and GAP (Goldberg, 1999; Lee et al., 2005b). Thus, an important future goal will be to pursue structural studies of these interactions, as they have the prospect of uncovering different ways by which the function of coatomer is regulated.

\section{MATERIALS AND METHODS}

\section{Antibodies}

For Western blotting, the rabbit polyclonal antibody specific for C-terminus of $\beta$-COP $(\mathrm{H}-300)$ was purchased from Santa Cruz Biotechnology. The anti-ARF1 antibody was raised against human $\operatorname{ARF} 1(\Delta N 17)$ in rabbits.

\section{Molecular cloning, protein expression, and purification}

The following cDNAs encoding for subunits of coatomer were synthesized commercially (Genewitz): $\alpha$-COP isoform 1 (accession number NM_001098398.1), $\beta$-COP (accession NM_001144061.1), $\beta^{\prime}$-COP (accession number NM_004766.2), Y-COP (accession number NM_016128.3), $\delta$-COP isoform 1 (accession number NM_001655.4), $\varepsilon$-COP isofom 1 (accession number NM_007263.3), and $\zeta$-COP isoform 1 (accession number NM_016057.2). The large plasmid containing all seven genes separated into two independent expression cassettes was developed by using ViperTEC method (to be published elsewhere) and transformed into $\mathrm{DH} 10 \mathrm{Bac}$ competent $E$. coli for bacmid generation. The presence of all seven subunits of coatomer in the bacmid was verified by PCR before transfecting into Sf9 insect cells. The generation of baculovirus was performed by standard procedures. All Sf9 cells were cultured in serum free media (either Sf900-II, Life Technologies or ESF921, Expression Systems).

For protein expression, we infected $600 \mathrm{~mL}$ spinner flask cultures of Sf9 cell at a density of $2 \times 10^{6}$ cells $/ \mathrm{mL}$ with $5 \mathrm{~mL}$ of P2 virus. After $72 \mathrm{~h}$, cells were harvested by centrifugation and suspended in cold lysis buffer $(50 \mathrm{mmol} / \mathrm{L}$ Tris- $\mathrm{Cl}, \mathrm{pH} 8.0,100 \mathrm{mmol} / \mathrm{L} \mathrm{KCl}, 10 \%$ glycerol, $1 \mathrm{mmol} / \mathrm{L}$ DTT) supplemented with protease inhibitors (Cocktail, complete-EDTA free, Roche Applied Science). Cells were lysed by ultra-sonication. After centrifugation, the cleared lysate was incubated with $1.5 \mathrm{~mL}$ pre-equilibrated Strep-Tactin Superflow beads (IBA). After washing with $50 \mathrm{~mL}$ lysis buffer, coatomer complex was eluted with $5 \mathrm{~mL}$ elution buffer (lysis buffer supplied with $5 \mathrm{mmol} / \mathrm{L}$ desthiobiotin). The elution was then loaded onto a $1 \mathrm{~mL}$ MonoQ anion exchange column (GE healthcare) pre-equilibrated with buffer A (20 mmol/L Bicine, pH 8.0, $100 \mathrm{mmol} / \mathrm{L} \mathrm{KCl,} 10 \%$ glycerol, $1 \mathrm{mmol} / \mathrm{L}$ DTT) and eluted using a gradient from $100 \mathrm{mmol} / \mathrm{L}$ to $1 \mathrm{~mol} / \mathrm{L} \mathrm{KCl}$ with buffer $\mathrm{B}(20 \mathrm{mmol} / \mathrm{L}$ Bicine, $\mathrm{pH} 8.0,1 \mathrm{~mol} / \mathrm{L} \mathrm{KCl}, 10 \%$ glycerol, $1 \mathrm{mmol} / \mathrm{L} \mathrm{DTT}$ ). Pooled fractions containing coatomer (approximately $0.35-0.4 \mathrm{~mol} / \mathrm{L} \mathrm{KCl}$ ) were concentrated and then diluted in order for the final $\mathrm{KCl}$ concentration to be approximately $100 \mathrm{mmol} / \mathrm{L}$. The twostep chromatography purification yielded above $90 \%$ pure coatomer at a protein concentration of $\sim 10 \mathrm{mg} / \mathrm{mL}$.

Recombinant human N-myristoylated Arf1 was purified from E. coli as previously described (Franco et al., 1996). Rat ArfGAP1 with a 10x His tag was expressed using BacMagic system and purified by affinity chromatography using Ni-NTA resin (GE Healthcare) and eluted by McAc300 buffer (50 mmol/L Tris-Cl, $\mathrm{pH} 8.0$, $300 \mathrm{mmol} / \mathrm{L} \mathrm{KCl}, 300 \mathrm{mmol} / \mathrm{L}$ imidazole).

\section{LC-MS protein identification}

The gel band of interest was manually excised from native PAGE gel and then digested following standard procedures. The digested solution was then transferred to a sample vial for LC-MS analysis. 
LC-MS analysis was performed using a Thermo Finnigan LTQ linear ion trap mass spectrometer in line with a Thermo Finnigan Surveyor MS Pump Plus HPLC system. Peptides generated above were loaded onto a trap column (300SB-C18, $5 \times 0.3 \mathrm{~mm}, 5 \mu \mathrm{m}$ particle) (Agilent Technologies, Santa Clara CA) which was connected through a zero dead volume union to the self-packed analytical column (C18, $100 \mu \mathrm{m}$ i.d $\times 100 \mathrm{~mm}, 3 \mu \mathrm{m}$ particle) (SunChrom, Germany). The peptides were then eluted over a gradient $(0 \%-45 \%$ $\mathrm{B}$ in $55 \mathrm{~min}, 45 \%-100 \% \mathrm{~B}$ in $10 \mathrm{~min}$, where $\mathrm{B}=80 \%$ Acetonitrile, $0.1 \%$ formic acid) at a flow rate of $500 \mathrm{~nL} / \mathrm{min}$ and introduced online into the linear ion trap mass spectrometer (ThermoFisher Corporation, San Jose, CA) using nano electrospray ionization (ESI). Data dependent scanning was incorporated to select the 5 most abundant ions (one microscan per spectra; precursor isolation width $1.0 \mathrm{~m} / \mathrm{z}$, $35 \%$ collision energy, $30 \mathrm{~ms}$ ion activation, exclusion duration: $90 \mathrm{~s}$; repeat count: 1) from a full-scan mass spectrum for fragmentation by collision induced dissociation (CID). MS data were analyzed using SEQUEST against NCBI human protein database; and results were filtered, sorted, and displayed using the Bioworks 3.2. Peptides with $+1,+2$, or +3 charge states were accepted if they were fully enzymatic and had a cross correlation (Xcorr) of $1.90,>2.5$, and $>3.0$, respectively. The following residue modifications were allowed in the search: carbamidomethylation on cysteine and oxidation on methionine. Sequest was searched with a peptide tolerance of 3 Amu and a fragment ion tolerance of $1.0 \mathrm{Amu}$.

\section{Preparations of liposomes}

Pure lipids were purchased from Avanti Polar lipids. Lipids were dissolved and mixed in either chloroform or methanol with composition similar to Golgi membrane (van Meer, 1998; van Meer et al., 2008): $45 \mathrm{~mol} \%$ DOPC, $19 \mathrm{~mol} \%$ DOPE, $5 \mathrm{~mol} \%$ DOPS, $8 \mathrm{~mol} \% \mathrm{PI}$, $7 \mathrm{~mol} \% \mathrm{SM}, 16 \mathrm{~mol} \%$ cholesterol. After evaporation, the dry lipid film was suspended and hydrated in Assay buffer $(25 \mathrm{mmol} / \mathrm{L}$ HEPES, $\left.\mathrm{pH} 7.2,25 \mathrm{mmol} / \mathrm{L} \mathrm{KCl}, 2.5 \mathrm{mmol} / \mathrm{L} \mathrm{Mg}(\mathrm{OAc})_{2}\right)$. After vortexing and repeated freezing/thawing, liposomes were further extruded through polycarbonate filter with $0.2 \mu \mathrm{m}$ pore.

\section{Membrane binding and vesiculation assays}

Incubation was carried out in $50 \mu \mathrm{L}$ AS buffer $(25 \mathrm{mmol} / \mathrm{L}$ Hepes, $\mathrm{pH}$ 7.2, $25 \mathrm{mmol} / \mathrm{L} \mathrm{KCl}, 0.2 \mathrm{~mol} / \mathrm{L}$ sucrose, $\left.2.5 \mathrm{mmol} / \mathrm{L} \mathrm{Mg}(\mathrm{OAc})_{2}\right)$ for $30 \mathrm{~min}$ at $37^{\circ} \mathrm{C}$ with myr-Arf1 $(4.8 \mu \mathrm{mol} / \mathrm{L})$, coatomer $(182 \mathrm{nmol} / \mathrm{L})$, GDP ( $1 \mathrm{mmol} / \mathrm{L}) / \mathrm{GTPYS}(25 \mu \mathrm{mol} / \mathrm{L})$, and liposome $(0.2 \mathrm{mg} / \mathrm{mL})$. EDTA ( $5 \mathrm{mmol} / \mathrm{L})$ was added firstly for guanine nucleotides exchange of Arf1, and then $\mathrm{Mg}(\mathrm{OAc})_{2}(10 \mathrm{mmol} / \mathrm{L})$ was supplied followed by the addition of other proteins into the incubation reactions. For studies of ArfGAP1, these incubation was carried out for $25 \mathrm{~min}$ at $37^{\circ} \mathrm{C}$ followed by additional incubation with ArfGAP1 $(1 \mu \mathrm{mol} / \mathrm{L})$ for $5 \mathrm{~min}$ at $37^{\circ} \mathrm{C}$. After incubation, $10 \mu \mathrm{L}$ of the reaction mix was reserved for negative staining and EM observation, and the remaining $40 \mu \mathrm{L}$ reaction mix was ultracentrifuged $(250,000 \times \mathrm{g}$, $30 \mathrm{~min}, 4^{\circ} \mathrm{C}$ ) through $500 \mu \mathrm{L}$ AS buffer to pellet membranes. After resuspending with AS buffer, the membranes and their bound proteins were analyzed by SDS-PAGE and Western blotting.

For purification of COPI-coated vesicles, the incubation volume was scaled up to $250 \mu \mathrm{L}$ with liposome at a concentration of $1.5 \mathrm{mg} / \mathrm{mL}$. After incubation, the reconstituted COPI vesicles were isolated from membranes and soluble proteins by subsequent centrifugation through two sucrose cushions in AS buffer $(37.5 \%, 50 \mu \mathrm{L} ; 45 \%, 10 \mu \mathrm{L})$, essentially as previously described (Beck et al., 2009). After centrifugation for $50 \mathrm{~min}$ at $100,000 \times \mathrm{g}$, the fraction at the interface of $37.5 \% /$ $45 \%$ sucrose cushion was collected and equally divided into two aliquots for either Western blotting or negative stain EM analysis.

\section{Negative stain electron microscopy}

For soluble coatomer, holy carbon grids coated with thin $(5 \mathrm{~nm})$ continuous carbon films, which was manufactured in-house, were used. Samples were applied to the glow-discharged grids and adsorbed for $1 \mathrm{~min}$ at RT. After washing with buffer, the grids were stained with $2 \%(w / v)$ uranyl acetate for $1 \mathrm{~min}$ at RT. For liposomes and vesicles, the incubation reaction solutions were directly applied onto glow-discharged EM grids (LifeTrust) coated with continuous carbon film and stained with $2 \%(w / v)$ uranyl acetate for $1 \mathrm{~min}$ at RT.

Images were collected on a $200 \mathrm{kV}$ FEI F200C Talos field emission transmission electron microscopy equipped with a $4 \mathrm{k} \times 4 \mathrm{~K}$ Ceta charge-coupled device camera (FEI, Netherlands). Single particle data were collected automatically with SerialEM software package (Mastronarde, 2005) at the magnification of 57,000 with a pixel size of $1.81 \AA$.

\section{Electron tomography and sub-tomogram averaging}

The same grid from the single particle data collection was applied for tilt series tomography data collection. Data were collected using SerialEM software package (Mastronarde, 2005) at the magnification of 57,000 with a pixel size of $1.81 \AA$. The angular range was from $-55^{\circ}$ to $+55^{\circ}$ with an increment of $5^{\circ}$ and the total electron dose was $80-100 \mathrm{e} / \AA^{2}$. Processing was performed on binned data with a pixel size of $3.6 \AA$. Gctf (Zhang, 2016) was used to estimate the defocus, which ranges from 5.0 $\mu \mathrm{m}$ to $8.0 \mu \mathrm{m}$. The tilt series were aligned using MarkerAuto (Han et al., 2015) before reconstruction using IMOD (Kremer et al., 1996). 632 subvolumes with a box size of 84 pixels were picked using IMOD (Kremer et al., 1996) and extracted using RELION1.4 (Bharat et al., 2015). Subsequently, sub-volume alignment and refinement were performed using RELION1.4 without CTF model. The initial model used in RELION1.4 refinement was a random rotational average of all the extracted sub-volumes (Bharat et al., 2015). The reconstruction after refinement was used as an initial model for the further single particle data processing.

\section{Single particle analysis}

Micrographs were screened out due to severe astigmatism, drift, inappropriate defocus, contamination, particle aggregation, and imperfect staining. Particles were first manually picked from 20 micrographs and class averaged using RELION1.3 (Scheres, 2012). Next, the class average results were used for template-based particle autopicking by Gautomatch (by Kai Zhang, MRC). To limit model bias, we applied a $40 \AA$ low-pass filter to these templates. An initial dataset of 353,777 particles was submitted to multiple cycles of reference-free 2D classification in RELION1.3 to exclude bad particles, such as contamination or overlapping particles. The 109,342 selected particles were used for 3D reconstruction with the 
initial model that was obtained from sub-tomogram average and filtered to $60 \AA$. After several cycles of 3D classification and refinement, a $2 \mathrm{D}$ classification step using the alignment parameters from the $3 \mathrm{D}$ refinement was performed to check the alignment accuracy and to further exclude particles liable to misalignment. The resulting 60,007 qualified particles were used for final 3D refinement and the subsequent "focus" refinement (Bai et al., 2015). For "focus" refinement, we segmented the final reconstruction map using the "Segmentation" tool in UCSF Chimera (Pettersen et al., 2004). The segmented densities were grouped into two sets, which were assigned as the two subcomplexes according to the rigid-body fitting of the membrane-bound coatomer structure. Then, the grouped densities were further extracted to a new volume data, and used for subtraction of the corresponding signals from the raw particles images using "relion_project" commands. The resulting new particle stacks were used as input in the subsequent $3 \mathrm{D}$ auto refine and classification processes.

\section{CXMS}

CXMS analysis was performed essentially as described previously (Ding et al., 2016). Briefly, in each $20 \mu \mathrm{L}$ cross-linking reaction, about $12 \mu \mathrm{g}$ of the purified COPI complex was incubated with $\mathrm{BS}^{3}$, DSS, or Sulfo-GMBS at a molar ratio of 1:8 or 1:4 (protein : cross-linker) for one hour at room temperature. The reactions were quenched with $20 \mathrm{mmol} / \mathrm{L} \mathrm{NH}_{4} \mathrm{HCO}_{3}$ for $\mathrm{BS}^{3}$ and DSS reactions, or with $20 \mathrm{mmol} / \mathrm{L}$ DTT, $20 \mathrm{mmol} / \mathrm{L} \mathrm{NH}_{4} \mathrm{HCO}_{3}$ for Sulfo-GMBS cross-linking. Proteins were precipitated with ice-cold acetone, resuspended in $8 \mathrm{~mol} / \mathrm{L}$ urea, $100 \mathrm{mmol} / \mathrm{L}$ Tris, $\mathrm{pH}$ 8.5. After Trypsin digestion, the LC-MS/ MS analysis was performed on an Easy-nLC 1000 UPLC (Thermo Fisher Scientific) coupled to a $Q$ Exactive-Orbitrap mass spectrometer (Thermo Fisher Scientific). Peptides were loaded onto a precolumn (75 $\mu \mathrm{m}$ ID, $8 \mathrm{~cm}$ long, packed with ODS-AQ $12 \mathrm{~nm}-10 \mu \mathrm{m}$ beads from YMC Co., Ltd.) and separated on an analytical column (75 $\mu \mathrm{m}$ ID, $11 \mathrm{~cm}$ long, packed with Luna C18 $1.8 \mu \mathrm{m} 100 \AA$ resin from Welch Materials) using an acetonitrile gradient from $0 \%-21 \%$ in $83 \mathrm{~min}$ at a flow rate of $200 \mathrm{~nL} / \mathrm{min}$. The top 15 most intense precursor ions from each full scan (resolution $=70,000$ ) were isolated for HCD MS2 (resolution = 17,500; normalized collisional energy = 27 ) with a dynamic exclusion time of $20 \mathrm{~s}$. Precursors with $1+, 2+, 7+$ or above, or unassigned charge states were excluded. Cross-linked peptides were identified using pLink (Yang et al., 2012) with a 5\% FDR cutoff at the spectral level and then an $E$-value cutoff at 0.001 .

The $\mathrm{Ca}-\mathrm{Ca}$ distance between two cross-linked residues were measured in PyMOL (The PyMOL Molecular Graphics System, Version 1.74, Schrodinger, LLC.). For the three structural models of clathrin AP-1 homologues, we first built models with human coatomer sequence, taking the crystal structure models (PDB ID: 4P6ZI $4 \mathrm{HMY} / 1 \mathrm{~W} 63$ ) as reference using Modeller software (Šali et al., 1995), and then the Ca-Ca distance between cross-linked residues were measured in PyMOL.

\section{ACKNOWLEDGMENTS}

We would like to thank Dr. Yan Zhang (F.S. lab) for help with image processing. We also thank Dr. Xiaojun Huang, Dr. Gang Ji, and Shuoguo Li from Center for Biological Imaging, Institute of Biophysics, CAS, for their help with EM data collection, and Dr. Xiang
Ding from Laboratory of Proteomics, Institute of Biophysics, CAS, for her help with the mass spectrometry analysis. This work was supported by grants from the Strategic Priority Research Program of Chinese Academy of Sciences (XDB08030202) to F.S., the National Basic Research Program (973 Program) (No. 2014CB910700 to F.S.,2014CB849800 to M.-Q.D.), the National Natural Science Foundation of China (Grant No. 21375010 to M.-Q.D.), and the National Institutes of Health USA (GM058651 to V.W.H.). All the EM work as well as related image processing were performed at Center for Biological Imaging (CBI, http://cbi.ibp.ac.cn), Institute of Biophysics, Chinese Academy of Sciences.

\section{ABBREVIATIONS}

ARF, ADP-ribosylation factor; COPI, coat protein I; CXMS, chemical cross-linking coupled with mass spectrometry; EM, electron microscopy; GAP, GTPase-activating protein; LC-MS, liquid chromatography mass spectrometry; NTD, N-terminal domain; CTD, C-terminal domain; PIP2, phosphatidylinositol $(4,5)$ bis-phosphate.

\section{COMPLIANCE WITH ETHICS GUIDELINES}

Shengliu Wang, Yujia Zhai, Xiaoyun Pang, Tongxin Niu, Yue-He Ding, Meng-Qiu Dong, Victor W. Hsu, Zhe Sun, and Fei Sun declare that they have no conflict of interest.

All procedures followed were in accordance with the ethical standards of the responsible committee on human experimentation (institutional and national) and with the Helsinki Declaration of 1975 , as revised in 2000 (5). Informed consent was obtained from all patients for being included in the study.

\section{AUTHOR CONTRIBUTIONS}

FS and ZS supervised the project. YZ performed molecular cloning and protein expression. SW performed protein expression and purification and all the EM works. SW, XP, and ZS performed functional studies. TN performed tomography works. YD and MD performed cross-linking and mass spectrometry. SW, ZS, and FS analyzed the data and wrote the manuscript with help from $\mathrm{VWH}$.

\section{OPEN ACCESS}

This article is distributed under the terms of the Creative Commons Attribution 4.0 International License (http://creativecommons.org/ licenses/by/4.0/), which permits unrestricted use, distribution, and reproduction in any medium, provided you give appropriate credit to the original author(s) and the source, provide a link to the Creative Commons license, and indicate if changes were made.

\section{REFERENCES}

Bai XC, Rajendra E, Yang GH, Shi YG, Scheres SHW (2015) Sampling the conformational space of the catalytic subunit of human gamma-secretase. eLife 4 
Beck R, Sun Z, Adolf F, Rutz C, Bassler J, Wild K, Sinning I, Hurt E, Brugger B, Bethune J et al (2008) Membrane curvature induced by Arf1-GTP is essential for vesicle formation. Proc Natl Acad Sci USA 105:11731-11736

Beck R, Adolf F, Weimer C, Bruegger B, Wieland FT (2009) ArfGAP1 Activity and COPI Vesicle Biogenesis. Traffic 10:307-315

Bharat TA, Russo CJ, Lowe J, Passmore LA, Scheres SH (2015) Advances in single-particle electron cryomicroscopy structure determination applied to sub-tomogram averaging. Structure 23:1743-1753

Bielli A, Haney CJ, Gabreski G, Watkins SC, Bannykh SI, Aridor M (2005) Regulation of Sar1 $\mathrm{NH}_{2}$ terminus by GTP binding and hydrolysis promotes membrane deformation to control COPII vesicle fission. J Cell Biol 171:919-924

Bigay J, Gounon P, Robineau S, Antonny B (2003) Lipid packing sensed by ArfGAP1 couples COPI coat disassembly to membrane bilayer curvature. Nature 426:563-566

Bonifacino JS, Glick BS (2004) The mechanisms of vesicle budding and fusion. Cell 116:153-166

Bremser M, Nickel W, Schweikert M, Ravazzola M, Amherdt M, Hughes CA, Sollner TH, Rothman JE, Wieland FT (1999) Coupling of coat assembly and vesicle budding to packaging of putative cargo receptors. Cell 96:495-506

Brugger B, Sandhoff R, Wegehingel S, Gorgas K, Malsam J, Helms JB, Lehmann WD, Nickel W, Wieland FT (2000) Evidence for segregation of sphingomyelin and cholesterol during formation of COPI-coated vesicles. J cell biol 151:507-518

Collins BM, McCoy AJ, Kent HM, Evans PR, Owen DJ (2002) Molecular architecture and functional model of the endocytic AP2 complex. Cell 109:523-535

Cosson P, Letourneur F (1994) Coatomer interaction with di-lysine endoplasmic reticulum retention motifs. Science 263:1629-1631

Cukierman E, Huber I, Rotman M, Cassel D (1995) The ARF1GTPase-activating protein: Zinc finger motif and Golgi complex localization. Science 270:1999-2002

Deng Y, Golinelli-Cohen MP, Smirnova E, Jackson CL (2009) A COPI coat subunit interacts directly with an early-Golgi localized Arf exchange factor. EMBO Rep 10:58-64

Ding Y, Fan S, Li S, Feng B, Gao N, Ye K, He S-M, Dong M-Q (2016) Increasing the depth of mass spectrometry-based structural analysis of protein complexes through the use of multiple crosslinkers. Anal Chem 88(8):4461-4469

Dodonova SO, Diestelkoetter-Bachert P, von Appen A, Hagen WJ, Beck R, Beck M, Wieland F, Briggs JA (2015) VESICULAR TRANSPORT. A structure of the COPI coat and the role of coat proteins in membrane vesicle assembly. Science 349:195-198

Donaldson JG, Cassel D, Kahn RA, Klausner RD (1992) ADPribosylation factor, a small GTP-binding protein, is required for binding of the coatomer protein beta-COP to Golgi membranes. Proc Natl Acad Sci USA 89:6408-6412

Faini M, Prinz S, Beck R, Schorb M, Riches JD, Bacia K, Brugger B, Wieland FT, Briggs JA (2012) The structures of COPI-coated vesicles reveal alternate coatomer conformations and interactions. Science 336:1451-1454

Faini M, Beck R, Wieland FT, Briggs JA (2013) Vesicle coats: structure, function, and general principles of assembly. Trends Cell Biol 23:279-288
Farsad K, Ringstad N, Takei K, Floyd SR, Rose K, De Camilli P (2001) Generation of high curvature membranes mediated by direct endophilin bilayer interactions. J Cell Biol 155:193-200

Fiedler K, Veit M, Stamnes MA, Rothman JE (1996) Bimodal interaction of coatomer with the p24 family of putative cargo receptors. Science 273:1396-1399

Franco M, Chardin P, Chabre M, Paris S (1996) Myristoylationfacilitated binding of the $G$ protein ARF1(GDP) to membrane phospholipids is required for its activation by a soluble nucleotide exchange factor. J Biol Chem 271:1573-1578

Goldberg J (1999) Structural and functional analysis of the ARF1ARFGAP complex reveals a role for coatomer in GTP hydrolysis. Cell 96:893-902

Han R, Wang L, Liu Z, Sun F, Zhang F (2015) A novel fully automatic scheme for fiducial marker-based alignment in electron tomography. J Struct Biol 192:403-417

Hara-Kuge S, Kuge O, Orci L, Amherdt M, Ravazzola M, Wieland FT, Rothman JE (1994) En bloc incorporation of coatomer subunits during the assembly of COP-coated vesicles [published erratum appears in J Cell Biol 1994 Jul; 126(2):589]. J Cell Biol 124:883-892

Hariri H, Bhattacharya N, Johnson K, Noble AJ, Stagg SM (2014) Insights into the mechanisms of membrane curvature and vesicle scission by the small GTPase Sar1 in the early secretory pathway. J Mol Biol 426:3811-3826

Heldwein EE, Macia E, Wang J, Yin HL, Kirchhausen T, Harrison SC (2004) Crystal structure of the clathrin adaptor protein 1 core. Proc Natl Acad Sci USA 101:14108-14113

Hsu VW, Lee SY, Yang JS (2009) The evolving understanding of COPI vesicle formation. Nat Rev Mol Cell Biol 10:360-364

Jackson LP (2014) Structure and mechanism of COPI vesicle biogenesis. Curr Opin Cell Biol 29:67-73

Jackson LP, Kelly BT, McCoy AJ, Gaffry T, James LC, Collins BM, Honing S, Evans PR, Owen DJ (2010) A large-scale conformational change couples membrane recruitment to cargo binding in the AP2 clathrin adaptor complex. Cell 141:1220-1229

Jackson LP, Lewis M, Kent HM, Edeling MA, Evans PR, Duden R, Owen DJ (2012) Molecular basis for recognition of dilysine trafficking motifs by COPI. Dev Cell 23:1255-1262

Jia, X., Weber, E., Tokarev, A., Lewinski, M., Rizk, M., Suarez, M., Guatelli, J., and Xiong, Y. (2014). Structural basis of HIV-1 Vpumediated BST2 antagonism via hijacking of the clathrin adaptor protein complex 1. eLife 3, e02362

Krauss M, Jia JY, Roux A, Beck R, Wieland FT, De Camilli P, Haucke $V$ (2008) Arf1-GTP-induced tubule formation suggests a function of Arf family proteins in curvature acquisition at sites of vesicle budding. J Biol Chem 283:27717-27723

Kremer JR, Mastronarde DN, McIntosh JR (1996) Computer visualization of three-dimensional image data using IMOD. J Struct Biol 116:71-76

Langer JD, Roth CM, Bethune J, Stoops EH, Brugger B, Herten DP, Wieland FT (2008) A conformational change in the alpha-subunit of coatomer induced by ligand binding to gamma-COP revealed by single-pair FRET. Traffic 9:597-607

Lee C, Goldberg J (2010) Structure of coatomer cage proteins and the relationship among COPI, COPII, and clathrin vesicle coats. Cell 142:123-132 
Lee MC, Orci L, Hamamoto S, Futai E, Ravazzola M, Schekman R (2005a) Sar1p N-terminal helix initiates membrane curvature and completes the fission of a COPII vesicle. Cell 122:605-617

Lee SY, Yang JS, Hong W, Premont RT, Hsu VW (2005b) ARFGAP1 plays a central role in coupling COPI cargo sorting with vesicle formation. J Cell Biol 168, 281-290

Leitner A, Walzthoeni T, Aebersold R (2014) Lysine-specific chemical cross-linking of protein complexes and identification of crosslinking sites using LC-MS/MS and the xQuest/xProphet software pipeline. Nat Protoc 9:120-137

Lundmark R, Doherty GJ, Vallis Y, Peter BJ, McMahon HT (2008) Arf family GTP loading is activated by, and generates, positive membrane curvature. Biochem J 414:189-194

Ma W, Goldberg J (2013) Rules for the recognition of dilysine retrieval motifs by coatomer. EMBO J 32:926-937

Malhotra V, Serafini T, Orci L, Shepherd JC, Rothman JE (1989) Purification of a novel class of coated vesicles mediating biosynthetic protein transport through the Golgi stack. Cell 58:329-336

Mastronarde DN (2005) Automated electron microscope tomography using robust prediction of specimen movements. J Struct Biol 152:36-51

Matsuoka K, Orci L, Amherdt M, Bednarek SY, Hamamoto S, Schekman R, Yeung T (1998) COPII-coated vesicle formation reconstituted with purified coat proteins and chemically defined liposomes. Cell 93:263-275

McMahon HT, Gallop JL (2005) Membrane curvature and mechanisms of dynamic cell membrane remodelling. Nature 438:590-596

Peter BJ, Kent HM, Mills IG, Vallis Y, Butler PJ, Evans PR, McMahon HT (2004) BAR domains as sensors of membrane curvature: the amphiphysin BAR structure. Science 303:495-499

Pettersen EF, Goddard TD, Huang CC, Couch GS, Greenblatt DM, Meng EC, Ferrin TE (2004) UCSF chimera - A visualization system for exploratory research and analysis. J Comput Chem 25:1605-1612

Politis A, Stengel F, Hall Z, Hernandez H, Leitner A, Walzthoeni T, Robinson CV, Aebersold R (2014) A mass spectrometry-based hybrid method for structural modeling of protein complexes. Nat Methods 11:403-406

Pucadyil TJ, Schmid SL (2009) Conserved functions of membrane active GTPases in coated vesicle formation. Science 325:12171220

Reinhard C, Harter C, Bremser M, Brugger B, Sohn K, Helms JB, Wieland $F$ (1999) Receptor-induced polymerization of coatomer. Proc Natl Acad Sci USA 96:1224-1228

Ren X, Farias GG, Canagarajah BJ, Bonifacino JS, Hurley JH (2013) Structural basis for recruitment and activation of the AP-1 clathrin adaptor complex by Arf1. Cell 152:755-767

Rosenthal PB, Henderson R (2003) Optimal determination of particle orientation, absolute hand, and contrast loss in single-particle electron cryomicroscopy. J Mol Biol 333:721-745

Sahlmuller MC, Strating JR, Beck R, Eckert P, Popoff V, Haag M, Hellwig A, Berger I, Brugger B, Wieland FT (2011) Recombinant heptameric coatomer complexes: novel tools to study isoformspecific functions. Traffic 12:682-692
Šali A, Potterton L, Yuan F, van Vlijmen H, Karplus M (1995) Evaluation of comparative protein modeling by MODELLER. Proteins: Structure. Funct Bioinform 23:318-326

Scheres SHW (2012) RELION: Implementation of a Bayesian approach to cryo-EM structure determination. J Struct Biol 180:519-530

Serafini T, Orci L, Amherdt M, Brunner M, Kahn RA, Rothman JE (1991a) ADP-ribosylation factor is a subunit of the coat of Golgiderived COP-coated vesicles: a novel role for a GTP-binding protein. Cell 67:239-253

Serafini T, Stenbeck G, Brecht A, Lottspeich F, Orci L, Rothman JE, Wieland FT (1991b) A coat subunit of Golgi-derived non-clathrincoated vesicles with homology to the clathrin-coated vesicle coat protein beta-adaptin. Nature 349:215-220

Shan H, Wang Z, Zhang F, Xiong Y, Yin CC, Sun F (2016) A localoptimization refinement algorithm in single particle analysis for macromolecular complex with multiple rigid modules. Protein Cell 7:46-62

Spang A, Matsuoka K, Hamamoto S, Schekman R, Orci L (1998) Coatomer, Arf1p, and nucleotide are required to bud coat protein complex I-coated vesicles from large synthetic liposomes. Proc Natl Acad Sci USA 95:11199-11204

Suckling RJ, Poon PP, Travis SM, Majoul IV, Hughson FM, Evans PR, Duden R, Owen DJ (2015) Structural basis for the binding of tryptophan-based motifs by delta-COP. Proc Natl Acad Sci USA 112:14242-14247

Takei K, Haucke V, Slepnev V, Farsad K, Salazar M, Chen H, De Camilli P (1998) Generation of coated intermediates of clathrinmediated endocytosis on protein-free liposomes. Cell 94:131141

van Meer G (1998) Lipids of the Golgi membrane. Trends Cell Biol 8:29-33

van Meer G, Voelker DR, Feigenson GW (2008) Membrane lipids: where they are and how they behave. Nat Rev Mol Cell Biol 9:112-124

Waters MG, Serafini T, Rothman JE (1991) 'Coatomer': a cytosolic protein complex containing subunits of non-clathrin-coated Golgi transport vesicles. Nature 349:248-251

Yang JS, Lee SY, Gao M, Bourgoin S, Randazzo PA, Premont RT, Hsu VW (2002) ARFGAP1 promotes the formation of COPI vesicles, suggesting function as a component of the coat. $\mathrm{J}$ Cell Biol 159:69-78

Yang B, Wu YJ, Zhu M, Fan SB, Lin J, Zhang K, Li S, Chi H, Li YX, Chen HF et al (2012) Identification of cross-linked peptides from complex samples. Nat Methods 9:904-906

Yip CK, Walz T (2011) Molecular structure and flexibility of the yeast coatomer as revealed by electron microscopy. J Mol Biol 408:825-831

Yu X, Breitman M, Goldberg J (2012) A structure-based mechanism for Arf1-dependent recruitment of coatomer to membranes. Cell 148:530-542

Zhang K (2016) Gctf: Real-time CTF determination and correction. J Struct Biol 193:1-12 